\title{
Weighted quasisymmetric enumerator for generalized permutohedra
}

\author{
Vladimir Grujić ${ }^{1}\left[\right.$ Marko Pešović $^{2} \cdot$ Tanja Stojadinović $^{1}$ \\ Received: 15 May 2018 / Accepted: 23 January 2019 / Published online: 27 March 2019 \\ (c) Springer Science+Business Media, LLC, part of Springer Nature 2019
}

\begin{abstract}
We introduce a weighted quasisymmetric enumerator function associated with generalized permutohedra. It refines the Billera, Jia and Reiner quasisymmetric function which also includes the Stanley chromatic symmetric function. Besides that, it carries information of face numbers of generalized permutohedra. We consider more systematically the cases of nestohedra and matroid base polytopes.
\end{abstract}

Keywords Generalized permutohedron · Quasisymmetric function · Combinatorial Hopf algebra $\cdot \mathrm{f}$-polynomial

Mathematics Subject Classification Primary 05E05; Secondary 52B05 · 16T05

\section{Introduction}

For a generalized permutohedron $Q$, there is a quasisymmetric enumerator function $F(Q)$ introduced by Billera, Jia and Reiner in [4]. It enumerates positive integer lattice points $\omega=\left(\omega_{1}, \ldots, \omega_{n}\right) \in \mathbb{Z}_{+}^{n}$ which are $Q$-generic. It means that the weight

\footnotetext{
A part of this research was appeared as an extended abstract for The 29th International Conference on Formal Power Series and Algebraic Combinatorics, FPSAC 2017. This research was supported by the Ministry of Education, Science and Technological developments of Republic of Serbia, Grant No.174034.

$\bowtie$ Vladimir Grujić

vgrujic@matf.bg.ac.rs

Marko Pešović

mpesovic@grf.bg.ac.rs

Tanja Stojadinović

tanjas@matf.bg.ac.rs

1 Faculty of Mathematics, University of Belgrade, Studentski trg 16, 11000 Belgrade, Serbia

2 Faculty of Civil Engineering, University of Belgrade, Bulevar kralja Aleksandra 73, 11000 Belgrade, Serbia
} 
function $\omega^{\star}: Q \rightarrow \mathbb{R}$ defined by $\omega^{\star}(x)=\langle\omega, x\rangle$ has its maximum at a unique vertex $v$ of $Q$. That is

$$
F(Q)=\sum_{\omega-\text { generic }} \mathbf{x}_{\omega},
$$

where $\mathbf{x}_{\omega}=x_{\omega_{1}} x_{\omega_{2}} \ldots x_{\omega_{n}}$. The generalized permutohedra are introduced and extensively studied by Postnikov [17] and Postnikov, Reiner and Williams [18]. They are deformations of standard permutohedra obtained by moving facets in normal directions. The faces of the standard permutohedron $P e^{n-1}$ are labeled by flags $\mathcal{F}$ of subsets of the set $[n]$. A part of the rich combinatorial structure of a generalized permutohedron $Q$ is a certain statistic $\mathrm{rk}_{Q}$ on the face lattice of the standard permutohedron $P e^{n-1}$ which we call the $Q$-rank. The $Q$-rank of a face $\mathcal{F}$ of $P e^{n-1}$ is dimension of the face of $Q$ to which $\mathcal{F}$ is deformed. The normal fan of the standard permutohedron $\Sigma_{P e^{n-1}}$ is the braid arrangement fan. The space $\mathbb{R}^{n}$ is decomposed by braid cones $\sigma_{\mathcal{F}}$. Each positive integer vector $\omega \in \mathbb{Z}_{+}^{n}$ determines a unique flag $\mathcal{F}_{\omega}$ such that $\omega$ lies in the relative interior of the braid cone $\sigma_{\mathcal{F}_{\omega}}$. We define the following weighted enumerator function associated with a generalized permutohedron Q

$$
F_{q}(Q)=\sum_{\omega \in \mathbb{Z}_{+}^{n}} q^{\mathrm{rk}_{Q}\left(\mathcal{F}_{\omega}\right)} \mathbf{x}_{\omega} .
$$

The $Q$-rank of a face $\mathcal{F}$ is zero if it is deformed into a vertex of $Q$. Henceforth, $F_{q}(Q)$ specializes at $q=0$ to the Billera, Jia and Reiner quasisymmetric enumerator function $F_{0}(Q)=F(Q)$.

On the other hand, when a class of generalized permutohedra is specified, we obtain the well known combinatorial enumerators. The case of graphical zonotopes $Q=Z_{\Gamma}$ is studied in [13]. The quasisymmetric function $F_{q}\left(Z_{\Gamma}\right)$ is a $q$-refinement of the Stanley chromatic symmetric function $X_{\Gamma}$ of graphs

$$
F_{0}\left(Z_{\Gamma}\right)=X_{\Gamma}
$$

The case of nestohedra is studied in [12] for $q=0$ and for a $q$-analogue in [14]. For a subclass of graph-associahedra $Q=P_{\Gamma}$, the enumerator $F\left(P_{\Gamma}\right)$ produces a new quasisymmetric invariant of a graph $\Gamma$ with a nice behavior.

The both cases of graphical zonotopes and nestohedra have in common that corresponding enumerators $F(Q)$ coincide with universal morphisms from certain combinatorial Hopf algebras to quasisymmetric functions. In the case of graphical zonotopes, it is the chromatic Hopf algebra of graphs and in the case of nestohedra, it is the non-cocommutative Hopf algebra on building sets and its Hopf subalgebra of graphs. Billera, Jia and Reiner applied their enumerator $F(Q)$ in the case of matroid base polytopes $Q=P_{M}$. The invariant $F\left(P_{M}\right)$ also comes from a Hopf algebra, in this case of matroids which was firstly introduced by Schmitt [19].

A remarkable and unifying approach to Hopf monoid structures constructed on combinatorial objects that provide generalized permutohedra have been developed in the recently published paper by M. Aguiar and F. Ardila [1]. Combining with the universality of quasisymmetric functions in the category of combinatorial Hopf 
algebras [2] shows the naturality of the invariant $F_{q}(Q)$ in enumerative and algebraic combinatorics.

The paper is organized as follows. In Sect. 2, we review some necessary facts about combinatorics of standard and generalized permutohedra. In Sect. 3, we review basic facts about quasisymmetric functions. In Sect. 4, we introduce the quasisymmetric function $F_{q}(Q)$ and show that it contains information about $f$-vectors of generalized permutohedra. It may be regarded as a far-reaching illumination of the Stanley $(-1)$-color theorem about the number of acyclic orientations of a graph. The cases of nestohedra and graph-associahedra are considered in Sects. 5 and 6. In the subsequent sections, the case of matroid base polytopes is considered more thoroughly. In Sect. 7, we review some basic facts about combinatorics of matroid base polytopes and introduce the combinatorial Hopf algebra of matroids and its $q$-analogue. The case of uniform matroids is presented. Finally, in Sect. 8, some properties of the weighted quasisymmetric enumerator function of matroids are derived.

\section{Generalized permutohedra}

The symmetric group $S_{n}$ acts on the space $\mathbb{R}^{n}$ by permuting the coordinates. Recall that a $(n-1)$-dimensional permutohedron $P e^{n-1}$ is the convex hull of the orbit of a point with increasing coordinates $x_{1}<\ldots<x_{n}$

$$
P e^{n-1}=\operatorname{Conv}\left\{\left(x_{\omega(1)}, x_{\omega(2)} \ldots, x_{\omega(n)}\right) \mid \omega \in S_{n}\right\} .
$$

The braid arrangement is the arrangement of hyperplanes $\left\{x_{i}=x_{j}\right\}_{1 \leq i<j \leq n}$ in the space $\mathbb{R}^{n}$. The regions of the braid arrangement, called Weyl chambers, are labeled by permutations $\omega \in S_{n}$

$$
C_{\omega}:=\left\{x_{\omega(1)} \leq x_{\omega(2)} \leq \ldots \leq x_{\omega(n)}\right\} .
$$

The corresponding braid arrangement fan is the normal fan $\Sigma_{P e^{n-1}}$ of the permutohedron $P e^{n-1}$. The cones of the braid arrangement fan are called braid cones.

A flag of length $|\mathcal{F}|=k$ on the set $[n]=\{1, \ldots, n\}$ is a chain of subsets

$$
\mathcal{F}: \emptyset=F_{0} \subset F_{1} \subset \ldots \subset F_{k-1} \subset F_{k} \subset F_{k+1}=[n] .
$$

The type of a flag $\mathcal{F}$ is the following $(k+1)$-tuple of integers

$$
\operatorname{type}(\mathcal{F})=\left(\left|F_{1}\right|-\left|F_{0}\right|,\left|F_{2}\right|-\left|F_{1}\right|, \ldots,\left|F_{k+1}\right|-\left|F_{k}\right|\right) .
$$

The set of flags is ordered by refinements. We write $\mathcal{G} \preceq \mathcal{F}$ if $\mathcal{F}$ refines $\mathcal{G}$. There is an obvious order reversing one-to-one correspondence between the face lattice of the permutohedron $P e^{n-1}$ and the lattice of flags of $[n]$. With no abuse of notation, we denote a face of the permutohedron $P e^{n-1}$ by the corresponding flag $\mathcal{F}$. Then $\mathcal{G} \preceq \mathcal{F}$ if and only if $\mathcal{F} \subseteq \mathcal{G}$ as faces of $P e^{n-1}$. By this convention, we have that 
$\operatorname{dim} \mathcal{F}=n-|\mathcal{F}|-1$. For example, the facets correspond to flags $\emptyset \subset A \subset[n]$, while the vertices correspond to maximal flags.

The braid cone $\sigma_{\mathcal{F}}$ at the face $\mathcal{F}$ is determined by coordinate relations

$$
\left\{\begin{array}{l}
x_{p}=x_{q} \text { if } p, q \in F_{i+1} \backslash F_{i} \text { for some } i=0, \ldots, k \\
x_{p} \leq x_{q} \text { if } p \in F_{i} \backslash F_{i-1} \text { and } q \in F_{i+1} \backslash F_{i} \text { for some } i=1, \ldots, k
\end{array}\right.
$$

Note that $\operatorname{dim}\left(\sigma_{\mathcal{F}}\right)=|\mathcal{F}|$ and the relative interior $\sigma_{\mathcal{F}}^{\circ}$, given by strict inequalities in the second condition above, is homeomorphic to $\mathbb{R}^{|\mathcal{F}|}$. Conversely, the flag $\mathcal{F}$ can be reconstructed from the braid cone $\sigma_{\mathcal{F}}$ by setting $p \in F_{i+1} \backslash F_{i}$ and $q \in F_{j+1} \backslash F_{j}$ for some $0 \leq i<j \leq k$ whenever $x_{p}<x_{q}$ for all points in the relative interior $\sigma_{\mathcal{F}}^{\circ}$.

Definition 2.1 A convex polytope $Q$ is an $(n-1)$-dimensional generalized permutohedron if its normal fan $\Sigma_{Q}$ is coarser than the braid arrangement fan $\Sigma_{P e^{n-1}}$.

Generalized permutohedra are equivalently characterized as deformations of the standard permutohedron $P e^{n-1}$ by moving its vertices with keeping directions of edges. Thus any edge of a generalized permutohedron $Q$ lies in the direction of some $e_{i}-e_{j}$, where $e_{i}, i=1, \ldots, n$ are the standard basis vectors in $\mathbb{R}^{n}$. For equivalent descriptions of generalized permutohedra, see [18].

Definition 2.2 For an $(n-1)$-dimensional generalized permutohedron $Q$, there is a map of face lattices

$$
\pi_{Q}: L\left(P e^{n-1}\right) \rightarrow L(Q)
$$

determined by $\pi_{Q}(\mathcal{F})=G$ if and only if the relative interior of the braid cone $\sigma_{\mathcal{F}}^{\circ}$ is contained in the relative interior $\sigma_{G}^{\circ}$ of the normal cone at the face $G$ of $Q$.

\section{Proposition 2.3}

$$
\sum_{\mathcal{F}: \pi_{Q}(\mathcal{F})=G}(-1)^{|\mathcal{F}|}=(-1)^{n-\operatorname{dim}(G)-1}
$$

Proof Consider the collection of flags $\pi_{Q}^{-1}(G)=\left\{\mathcal{F}_{1}, \mathcal{F}_{2}, \ldots \mathcal{F}_{t}\right\}$. We have $\sigma_{G}^{\circ}=$ $\bigcup_{i=1}^{t} \sigma_{\mathcal{F}_{i}}^{\circ}$ and $\sigma_{G}^{\circ}$ is homeomorphic to the $k$-dimensional open cell, where $k=n-$ $\operatorname{dim} G-1$. Let $f_{i}, i=0,1, \ldots, k$ be the numbers of $i$-dimensional cones such that $\sigma_{\mathcal{F}}^{\circ} \subset \sigma_{G}^{\circ}$. By inclusion-exclusion principle we have

$$
f_{k}-f_{k-1}+\ldots+(-1)^{k} f_{0}=1 \text {. }
$$

\section{Quasisymmetric functions}

In this section, we review the basic facts about combinatorial Hopf algebras and quasisymmetric functions. The notion of combinatorial Hopf algebra, originated in the 
work of Aguiar, Bergeron and Sottile [2], gives a natural algebraic framework of enumerative combinatorics. The extensive survey of Hopf algebra theory in combinatorics may be found in [11].

A combinatorial Hopf algebra is a graded, connected Hopf algebra $\mathcal{H}$ equipped with a multiplicative linear functional $\zeta: \mathcal{H} \rightarrow \mathbf{k}$ to the ground field. We describe the combinatorial Hopf algebra of quasisymmetric functions $Q S y m$.

A quasisymmetric function $F=F(\mathbf{x})$ is a formal power series of bounded degree in the countable ordered set of variables $\mathbf{x}=\left(x_{1}, x_{2}, \ldots\right)$ such that coefficients by monomials with the same list of ordered exponents are equal. This condition produces a natural linear basis for the algebra $Q S y m$ consisting of monomial quasisymmetric functions

$$
M_{\alpha}=\sum_{i_{1}<i_{2}<\cdots<i_{k}} x_{i_{1}}^{a_{1}} x_{i_{2}}^{a_{2}} \ldots x_{i_{k}}^{a_{k}},
$$

indexed by finite ordered sets of integers $\alpha=\left(a_{1}, a_{2}, \ldots, a_{k}\right)$ called compositions of $|\alpha|=a_{1}+a_{2}+\cdots+a_{k}$ of length $k(\alpha)=k$. The coproduct, defined on the monomial basis by

$$
\Delta\left(M_{\alpha}\right)=\sum_{\beta \gamma=\alpha} M_{\beta} \otimes M_{\gamma},
$$

where $\beta \gamma$ is the concatenation of compositions, turns $Q S y m$ into a graded, connected Hopf algebra.

The principal specialization ps : $Q S y m \rightarrow \mathbf{k}[m]$ assigns to a quasisymmetric function $F$ a unique polynomial which agrees for each positive integer $m$ with the evaluation map

$$
\operatorname{ps}(F)(m)=\left.F\right|_{x_{1}=\cdots=x_{m}=1, x_{m+1}=\cdots=0} .
$$

The canonical character on quasisymmetric functions $\zeta_{\mathcal{Q}}: Q S y m \rightarrow \mathbf{k}$ is defined by

$$
\zeta_{\mathcal{Q}}(F)=\mathbf{p s}(F)(1)
$$

It is easy to see that on the monomial basis we have

$$
\operatorname{ps}\left(M_{\alpha}\right)(m)=\left(\begin{array}{c}
m \\
k(\alpha)
\end{array}\right)
$$

and specially

$$
\operatorname{ps}\left(M_{\alpha}\right)(-1)=(-1)^{k(\alpha)} .
$$

The following theorem is fundamental in applications and explains the ubiquity of quasisymmetric functions as enumerator functions in combinatorics. 
Theorem 3.1 ([2], Theorem 4.1) For a combinatorial Hopf algebra $(\mathcal{H}, \zeta)$ there is a unique morphism of graded Hopf algebras

$$
\Psi: \mathcal{H} \rightarrow Q S y m
$$

such that $\Psi \circ \zeta_{\mathcal{Q}}=\zeta$. For a homogeneous element $h$ of degree $n$ the coefficients $\zeta_{\alpha}(h), \alpha=\left(a_{1}, \ldots, a_{k}\right)$ of $\Psi(h)$ in the monomial basis of $Q S y m$ are given by

$$
\zeta_{\alpha}(h)=\zeta^{\otimes k} \circ\left(p_{a_{1}} \otimes p_{a_{2}} \otimes \cdots \otimes p_{a_{k}}\right) \circ \Delta^{(k-1)}(h),
$$

where $p_{i}$ is the projection on the $i$-th homogeneous component and $\Delta^{(k-1)}$ is the $(k-1)$-fold coproduct map of $\mathcal{H}$.

\section{Weighted quasisymmetric enumerator $F_{q}(Q)$}

A generalized permutohedron $Q$ comes with a natural map between face lattices $\pi_{Q}: L\left(P e^{n-1}\right) \rightarrow L(Q)$ given by Definition 2.2 with $\pi_{Q}(\mathcal{F})=G$ if and only if $\sigma_{\mathcal{F}}^{\circ} \subset \sigma_{G}^{\circ}$. This map produces a natural statistic of faces of the standard permutohedron $P e^{n-1}$.

Definition 4.1 For a generalized permutohedron $Q$ the $Q$-rank is a map on the face lattice of the standard permutohedron $\mathrm{rk}_{Q}: L\left(P e^{n-1}\right) \rightarrow\{0,1, \ldots, n-1\}$ given by

$$
\operatorname{rk}_{Q}(\mathcal{F})=\operatorname{dim}\left(\pi_{Q}(\mathcal{F})\right)
$$

Let $\omega=\left(\omega_{1}, \omega_{2}, \ldots, \omega_{n}\right) \in \mathbb{Z}_{+}^{n}$ be an integer lattice vector with positive entries. It defines the weight function $\omega^{\star}: Q \rightarrow \mathbb{R}$ on $Q$ by $\omega^{\star}(x)=\langle\omega, x\rangle$, where $\langle$,$\rangle is the$ standard scalar product in $\mathbb{R}^{n}$. Note that $Q$ lies in a hyperplane whose normal vector is $(1, \ldots, 1)$. The weight function $\omega^{\star}$ is maximized along a unique face $G_{\omega}$ of $Q$ which is determined by the condition that the vector $\omega$ lies in the relative interior of its normal cone $\omega \in \sigma_{G_{\omega}}^{\circ}$. A weight function $\omega^{\star}$ is called $Q$-generic if $G_{\omega}$ is a vertex of $Q$.

If $Q=P e^{n-1}$ is the standard permutohedron each $\omega \in \mathbb{Z}_{+}^{n}$ determines a unique flag $\mathcal{F}_{\omega}$ by the condition $\mathcal{F}=\mathcal{F}_{\omega}$ if and only if the integer vector $\omega$ lies in the relative interior of the corresponding braid cone $\omega \in \sigma_{\mathcal{F}}^{\circ}$.

Definition 4.2 For a generalized permutohedron $Q$ let $F_{q}(Q)$ be a weighted enumerator of positive integer vectors

$$
F_{q}(Q)=\sum_{\omega \in \mathbb{Z}_{+}^{n}} q^{\mathrm{rk}_{Q}\left(\mathcal{F}_{\omega}\right)} \mathbf{x}_{\omega} .
$$

We expand the enumerator function $F_{q}(Q)$ in the monomial basis of quasisymmetric functions. 
Definition 4.3 For a flag $\mathcal{F}$ of subsets of $[n]$ let $M_{\mathcal{F}}$ be the enumerator of positive integer vectors in the relative interior of the corresponding braid cone

$$
M_{\mathcal{F}}=\sum_{\omega \in \mathbb{Z}_{+}^{n} \cap \sigma_{\mathcal{F}}^{\circ}} \mathbf{x}_{\omega} .
$$

The enumerator $M_{\mathcal{F}}$ is exactly a monomial quasisymmetric function depending only on the type of $\mathcal{F}$, given by (2.1)

$$
M_{\mathcal{F}}=M_{\text {type }(\mathcal{F})} .
$$

We obtain an expansion of $F_{q}(Q)$ according to the face lattice of the standard permutohedron

$$
F_{q}(Q)=\sum_{\mathcal{F} \in L\left(P e^{n-1}\right)} q^{\mathrm{rk}_{Q}(\mathcal{F})} M_{\mathcal{F}} .
$$

In the monomial basis, $F_{q}(Q)$ has expansion of the form

$$
F_{q}(Q)=\sum_{\alpha \mid=n} p_{\alpha}(q) M_{\alpha}
$$

where $p_{\alpha}(q)$ are polynomials in $q$ indexed by compositions of $n$. For a composition $\alpha$, the polynomial $p_{\alpha}(q)$ is given by

$$
p_{\alpha}(q)=\sum_{\mathcal{F}: \operatorname{type}(\mathcal{F})=\alpha} q^{\mathrm{rk}_{Q}(\mathcal{F})} .
$$

By Definition 2.2 of the map $\pi_{Q}$, we have

$$
\sum_{\omega \in \sigma_{G}^{\circ}} \mathbf{x}_{\omega}=\sum_{\mathcal{F}: \pi_{Q}(\mathcal{F})=G} M_{\mathcal{F}}
$$

which gives an expansion of $F_{q}(Q)$ in terms of the face lattice of $Q$

$$
F_{q}(Q)=\sum_{G \in L(Q)} q^{\operatorname{dim}(G)} \sum_{\mathcal{F}: \pi_{Q}(\mathcal{F})=G} M_{\mathcal{F}}
$$

From this, we easily derive that the enumerator $F_{q}$ contains information about $f$ vectors of generalized permutohedra. The $f$-vector of a convex $(n-1)$-dimensional polytope $P$ is the integer vector $f=\left(f_{0}, f_{1}, \ldots, f_{n-1}\right)$, where $f_{i}$ is the number of $i$-dimensional faces of $P$. It is codified by the $f$-polynomial

$$
f(P, q)=f_{0}+f_{1} q+f_{2} q^{2}+\ldots+f_{n-1} q^{n-1} .
$$


Theorem 4.4 The $f$-polynomial $f(Q, q)$ of an $(n-1)$-dimensional generalized permutohedron $Q$ is determined by the principal specialization

$$
f(Q, q)=(-1)^{n} \mathbf{p s}\left(F_{-q}(Q)\right)(-1)
$$

Proof By formula (4.2) and the fact $\operatorname{ps}\left(M_{\mathcal{F}}\right)(-1)=(-1)^{|\mathcal{F}|+1}$ implied by (3.1) the principal specialization of $F_{q}(Q)$ gives

$$
(-1)^{n} \operatorname{ps}\left(F_{-q}(Q)\right)(-1)=\sum_{G \in L(Q)} q^{\operatorname{dim}(G)} \sum_{\mathcal{F}: \pi_{Q}(\mathcal{F})=G}(-1)^{|\mathcal{F}|+1+n+\operatorname{dim}(G)} .
$$

The inner sum is equal to 1 by Proposition 2.3, which completes the proof.

\subsection{Action of the antipode on $F_{q}(Q)$}

In this subsection, we determine how the antipode $S$ of the Hopf algebra of quasisymmetric functions $Q S y m$ acts on the weighted quasisymmetric enumerator function $F_{q}(Q)$.

Define the opposite flag $\mathcal{F}^{o p}$ to a flag $\mathcal{F}: \emptyset=F_{0} \subset F_{1} \subset \ldots \subset F_{k} \subset F_{k+1}=[n]$ by

$$
\mathcal{F}^{o p}: \emptyset \subset[n] \backslash F_{k} \subset \cdots \subset[n] \backslash F_{1} \subset[n]
$$

The corresponding braid cones are related by $\sigma_{\mathcal{F} o p}=-\sigma_{\mathcal{F}}$. For a composition $\alpha=$ $\left(a_{1}, \ldots, a_{k}\right)$, the reverse composition is $\operatorname{rev}(\alpha)=\left(a_{k}, \ldots, a_{1}\right)$. We have

$$
\operatorname{type}\left(\mathcal{F}^{o p}\right)=\operatorname{rev}(\operatorname{type}(\mathcal{F}))
$$

Recall that flags are ordered by refinements. The following lemma gives a particularly nice geometric meaning of the formula for the antipode $S$, see [11, Theorem 5.1.11].

Lemma 4.5 The antipode $S$ on the monomial quasisymmetric function $M_{\mathcal{F}}$ associated with a flag $\mathcal{F}$ acts by

$$
S\left(M_{\mathcal{F}}\right)=(-1)^{|\mathcal{F}|+1} \sum_{\mathcal{G} \preceq \mathcal{F} o p} M_{\mathcal{G}}
$$

This allows us to interpret $S\left(M_{\mathcal{F}}\right)$ as the enumerator function of integer lattice points lying in the opposite braid cone $\sigma_{\mathcal{F} o p}$

$$
(-1)^{|\mathcal{F}|+1} S\left(M_{\mathcal{F}}\right)=\sum_{\omega \in \mathbb{Z}_{+}^{n} \cap \sigma_{\mathcal{F}} o p} \mathbf{x}_{\omega}
$$

Let $\pi_{Q}$ be the map associated with a generalized permutohedron $Q$ by Definition 2.2. We say that the face $\pi_{Q}\left(\mathcal{F}^{o p}\right)$ is opposite to a flag $\mathcal{F}$. The following theorem 
describes the action of the antipode on the weighted quasisymmetric enumerator function $F_{q}(Q)$.

Theorem 4.6 Given a generalized permutohedron $Q$ of dimension $n-1$, the antipode $S$ acts on the weighted quasisymmetric enumerator function $F_{q}(Q)$ by

$$
S\left(F_{q}(Q)\right)=(-1)^{n} \sum_{\mathcal{G}} f\left(\pi_{Q}\left(\mathcal{G}^{o p}\right),-q\right) M_{\mathcal{G}},
$$

where the sum is over all flags $\mathcal{G}$ of the set $[n]$ and $f\left(\pi_{Q}\left(\mathcal{G}^{o p}\right), q\right)$ is the $f$-polynomial of the face $\pi_{Q}\left(\mathcal{G}^{o p}\right)$ opposite to a flag $\mathcal{G}$.

Proof By the expansion (4.1) and Lemma 4.5 we have

$$
S\left(F_{q}(Q)\right)=\sum_{\mathcal{F}} q^{\mathrm{rk}_{Q}(\mathcal{F})} S\left(M_{\mathcal{F}}\right)=\sum_{\mathcal{F}} q^{\mathrm{rk}_{Q}(\mathcal{F})}(-1)^{|\mathcal{F}|+1} \sum_{\mathcal{G} \preceq \mathcal{F} o p} M_{\mathcal{G}},
$$

which gives

$$
S\left(F_{q}(Q)\right)=\sum_{\mathcal{G}} M_{\mathcal{G}} \sum_{\mathcal{F}: \mathcal{G}^{o p} \preceq \mathcal{F}}(-1)^{|\mathcal{F}|+1} q^{\mathrm{rk}_{Q}(\mathcal{F})} .
$$

It remains to show that

$$
\sum_{\mathcal{F}: \mathcal{G}^{o p} \preceq \mathcal{F}}(-1)^{|\mathcal{F}|+1} q^{\mathrm{rk}_{Q}(\mathcal{F})}=(-1)^{n} f\left(\pi_{Q}\left(\mathcal{G}^{o p}\right),-q\right),
$$

i.e.

$$
f\left(\pi_{Q}\left(\mathcal{G}^{o p}\right), q\right)=\sum_{\mathcal{F}: \mathcal{G}^{o p} \preceq \mathcal{F}}(-1)^{|\mathcal{F}|+1+n+\mathrm{rk}_{Q}(\mathcal{F})} q^{\mathrm{rk}_{Q}(\mathcal{F})},
$$

which is a consequence of Definition 4.1 and Proposition 2.3.

Theorem 4.6 generalizes Theorem 4.4. To see this note that $\pi_{Q}(\emptyset \subset[n])=Q$ and by Theorem 4.6, the coefficient in $S\left(F_{q}(Q)\right)$ by the monomial quasisymmetric function $M_{(n)}=M_{\emptyset \subset[n]}$ is equal to $(-1)^{n} f(Q,-q)$. This coefficient can be extracted from $S\left(F_{q}(Q)\right)$ by composing with the canonical character $\zeta_{\mathcal{Q}}$ on quasisymmetric functions. Theorem 4.4 then follows from the fact

$$
\zeta_{\mathcal{Q}} \circ S(F)=\mathbf{p s}(F)(-1)
$$

which can be easily seen to hold for the monomial basis and consequently for each quasisymmetric function $F$.

Corollary 4.7 For a generalized permutohedron $Q$, the following identity holds

$$
\operatorname{ps}\left(S\left(F_{q}(Q)\right)\right)(-1)=q^{\operatorname{dim}(Q)}
$$


Proof It follows from the Eq. (4.4) and the fact that the antipode $S$ of $Q S y m$ is of order two $S^{2}=$ Id.

\subsection{Specializations of the enumerator $F_{q}(Q)$}

We review some specializations of the quasisymmetric enumerator function $F_{q}(Q)$. By taking $q=0$, it is specified to the Billera, Jia and Reiner quasisymmetric function $F(Q)$ which is the enumerator of $Q$-generic weight vectors

$$
F(Q)=\sum_{\omega \in \mathbb{Z}_{+}^{n}: \operatorname{rk}\left(\mathcal{F}_{\omega}\right)=0} \mathbf{x}_{\omega} .
$$

Theorem 4.4 may be regarded as a generalization of the formula for number of vertices $f_{0}$ of a generalized permutohedron $Q$, see [4, Theorem 9.2]

$$
f_{0}=(-1)^{n} \mathbf{p s}(F(Q))(-1)
$$

Note that $F_{q}(Q)$ degenerates at $q=1$ since $F_{1}(Q)=\sum_{\omega \in \mathbb{Z}_{+}^{n}} \mathbf{x}_{\omega}=\left(M_{(1)}\right)^{n}$. Theorem 4.4 then gives

$$
f(Q,-1)=f_{0}-f_{1}+\cdots+(-1)^{n-1} f_{n-1}=(-1)^{n} \mathbf{p s}\left(M_{(1)}^{n}\right)(-1)=1,
$$

which is the Euler characteristic of $Q$.

For particular classes of generalized permutohedra, the enumerator $F_{q}$ specializes to known quasisymmetric invariants as it is announced in introduction. We briefly review the case of graphical zonotopes and more thoroughly the cases of nestohedra and matroid base polytopes in subsequent sections.

Graphical zonotopes To a simple connected graph $\Gamma$ on $n$ vertices is associated a convex $(n-1)$-dimensional polytope $Z_{\Gamma}$ called graphical zonotope. The vertices of the graphical zonotope $Z_{\Gamma}$ are in one-to-one correspondence with regions of the corresponding graphical arrangement $\mathcal{H}_{\Gamma}=\left\{x_{i}=x_{j} \mid\{i, j\} \in E(\Gamma)\right\}$ whose fan is the normal fan of $Z_{\Gamma}$. The integer points $\omega \in \mathbb{Z}_{+}^{n}$ can be interpreted as graph colorings by labeling vertices of the graph $\Gamma$. A coloring is proper if there are no monochromatic edges. The normal cone at a vertex of $Z_{\Gamma}$ is a region of the corresponding graphical arrangement. So $\omega$ determines a proper coloring of the graph $\Gamma$ if and only if $\mathrm{rk}_{Z_{\Gamma}}\left(\mathcal{F}_{\omega}\right)=0$. Thus the enumerators of proper colorings and $Z_{\Gamma}$-generic integer vectors coincide, i.e. $X_{\Gamma}=F_{0}\left(Z_{\Gamma}\right)$, where $X_{\Gamma}$ is the Stanley chromatic symmetric function (introduced in [20]).

The main argument in [13] of the proof of Theorem 4.4 for graphical zonotopes was based on the Humpert and Martin cancelation-free formula for the antipode of the chromatic Hopf algebra of graphs [15]. 


\section{Nestohedra}

We refer the reader to [18] for definitions and main properties of nestohedra. The nestohedra are a class of simple polytopes described by the notion of building sets. A collection of subsets $B$ of a finite ground set $V$ is a building set if

- $\{i\} \in B$ for all $i \in V$ and

- if $I, J \in B$ and $I \cap J \neq \varnothing$ then $I \cup J \in B$.

A building set $B$ is connected if $V \in B$. Let $\Delta^{n-1}=\operatorname{Conv}\left\{e_{1}, \ldots, e_{n}\right\}$ be the standard simplex in $\mathbb{R}^{n}$. The nestohedron associated with a building set $B$ on the ground set $[n]$ is the Minkowski sum of simplices $P_{B}=\sum_{I \in B} \operatorname{Conv}\left\{e_{i} \mid i \in I\right\}$. Enumerate faces of $\Delta^{n-1}$ by subsets of $[n]$ in a way that the face poset of $\Delta^{n-1}$ is isomorphic to the reverse Boolean lattice on $[n]$. For a connected building set $B$, the nestohedron $P_{B}$ is realized by successive truncations over faces of $\Delta^{n-1}$ encoded by a building set $B$ in any nondecreasing sequence of dimensions of faces. Thus, facets of $P_{B}$ are labeled by elements $I \in B \backslash\{[n]\}$. Recall that a truncation of a convex polytope $P$ over a face $F \subset P$ is the polytope $P \backslash F$ obtained by cutting $P$ with a hyperplane $H_{F}$ which divides vertices in $F$ and vertices not in $F$ in separated halfspaces. For a disconnected building set $B$, the associated nestohedron $P_{B}$ is the product of nestohedra corresponding to components of $B$.

The following condition describes the face poset of a nestohedron $P_{B}$ corresponding to a connected building set $B$, see [8, Theorem 3.14] and [17, Theorem 7.4]. The intersection $F_{I_{1}} \cap \ldots \cap F_{I_{k}}, k \geq 2$ of facets corresponding to a subcollection $N=$ $\left\{I_{1}, \ldots, I_{k}\right\} \subset B \backslash\{[n]\}$ is a nonempty face of $P_{B}$ if and only if

(N1) $I_{i} \subset I_{j}$ or $I_{j} \subset I_{i}$ or $I_{i} \cap I_{j}=\emptyset$ for any $1 \leq i<j \leq k$,

(N2) $I_{j_{1}} \cup \cdots \cup I_{j_{p}} \notin B$ for any pairwise disjoint sets $I_{j_{1}}, \ldots, I_{j_{p}}$.

Subcollections that satisfy conditions (N1) and (N2) form a simplicial complex, called the nested set complex, whose face poset is opposite to the face poset of $P_{B}$.

\subsection{Hopf algebra $\mathcal{B}$}

Two building sets $B_{1}$ and $B_{2}$ are isomorphic if there is a bijection of their sets of vertices $f: V_{1} \rightarrow V_{2}$ such that $I \in B_{1}$ if and only if $f(I) \in B_{2}$. The addition of building sets $B_{1}$ and $B_{2}$ on disjoint ground sets $V_{1}$ and $V_{2}$ is the building set $B_{1} \sqcup B_{2}=$ $\left\{I \subset V_{1} \sqcup V_{2} \mid I \in B_{1}\right.$ or $\left.I \in B_{2}\right\}$. For a building set $B$ on $V$ and a subset $S \subset V$, the restriction on $S$ and the contraction of $S$ from $B$ are defined by $\left.B\right|_{S}=\{I \subset S \mid I \in B\}$ and $B / S=\left\{I \subset V \backslash S \mid I \in B\right.$ or $I \cup S^{\prime} \in B$ for some $\left.S^{\prime} \subset S\right\}$. The building sets obtained from $B$ by restrictions and contractions are its minors.

The following combinatorial Hopf algebra of building sets is considered in [12]. The set of all isomorphism classes of finite building sets linearly generates the vector space $\mathcal{B}$ over a field $\mathbf{k}$. The space $\mathcal{B}$ is a graded, commutative and non-cocommutative Hopf algebra with the product and the coproduct

$$
\left[B_{1}\right] \cdot\left[B_{2}\right]=\left[B_{1} \sqcup B_{2}\right] \text { and } \Delta([B])=\sum_{S \subset V}\left[\left.B\right|_{S}\right] \otimes[B / S]
$$


The grading $\operatorname{gr}([B])$ is given by cardinality of the ground set of $B$. A building set $B$ is connected if $[B]$ is irreducible, i.e., it cannot be represented by an addition of two building sets. Denote by $c(B)$ the number of connected components of $B$. Let $\zeta: \mathcal{B} \rightarrow \mathbf{k}$ be a multiplicative linear functional defined by $\zeta([B])=1$ if $B$ is a discrete (consisting of singletons only) and $\zeta([B])=0$ otherwise. By Theorem 3.1 a unique morphism $\Psi:(\mathcal{B}, \zeta) \rightarrow\left(Q S y m, \zeta_{Q}\right)$ is given in the monomial basis by

$$
\Psi([B])=\sum_{\alpha \models \operatorname{gr}(B)} \zeta_{\alpha}(B) M_{\alpha} .
$$

The coefficients $\zeta_{\alpha}(B)$ have an enumerative meaning. Let $\mathcal{L}: \emptyset \subset I_{1} \subset \cdots \subset I_{k}=V$ be a chain of subsets of the ground set $[n]$. Denote by $|\mathcal{L}|=k$ its length and by type $(\mathcal{L})$ its type which is a composition $\alpha=\left(i_{1}, \ldots, i_{k}\right)$ such that for any $1 \leq j \leq k$ the set $I_{j} \backslash I_{j-1}$ has $i_{j}$ elements. We say that $\mathcal{L}$ is a splitting chain if all minors $\left.B\right|_{I_{j}} / I_{j-1}$ are discrete. Then, $\zeta_{\alpha}(B)$ is exactly the number of splitting chains of $B$ of a given type $\alpha$. For a building set $B$ on $[n]$, the following identity holds ([12, Theorem 4.5])

$$
F\left(P_{B}\right)=\Psi([B]) .
$$

\section{2 q-analogue}

We extend the basic field $\mathbf{k}$ into the field of rational functions $\mathbf{k}(q)$ and define the character $\zeta_{q}: \mathcal{B} \rightarrow \mathbf{k}(q)$ with $\zeta_{q}([B])=q^{\operatorname{rk}(B)}$, where $\operatorname{rk}(B)=\operatorname{gr}(B)-c(B)$. Let $\Psi_{q}:\left(\mathcal{B}, \zeta_{q}\right) \rightarrow\left(Q \operatorname{Sym}, \zeta_{Q}\right)$ be a unique morphism of combinatorial Hopf algebras over $\mathbf{k}(q)$.

Recall that a reflexive and transitive relation $\preccurlyeq$ on $[n]$ is called a preorder. If in addition $p \preccurlyeq q$ or $q \preccurlyeq p$ for any $p, q \in[n]$ it is called a weak order on [ $n]$. Weak orders correspond to set compositions of $[n]$. Each set composition determines a unique flag of subsets and vice versa. We say that a flag of subsets $\mathcal{L}: \emptyset=I_{0} \subset I_{1} \subset \ldots \subset I_{k}=[n]$ extends a preorder $\preccurlyeq$ on $[n]$ if

$$
i \prec j \text { implies } i \in I_{p} \backslash I_{p-1} \text { and } j \in I_{q} \backslash I_{q-1} \text { for some } 1 \leq p<q \leq k \text {, }
$$

where $i \prec j$ means that $i \preccurlyeq j$ and it is not $j \preccurlyeq i$.

Let $G=F_{I_{1}} \cap \ldots \cap F_{I_{m}}$ be a face of $P_{B}$ and $N_{G}=\left\{I_{1}, \ldots, I_{m}\right\} \subset B \backslash\{[n]\}$ the corresponding nested set. Note that $\operatorname{dim} G=n-1-m$. For each $I \in N_{G} \cup\{[n]\}$ let $I^{\text {root }}$ be the set of roots of $I$ given by

$$
I^{\text {root }}=I \backslash \cup\left\{J \in N_{G} \mid J \varsubsetneqq I\right\} .
$$

Define a preorder $\preccurlyeq G$ on $[n]$ corresponding to the face $G \subset P_{B}$ by

$$
i \preccurlyeq \preccurlyeq_{G} j \text { if and only if } i \in I, j \in I^{\text {root }} \text { for some } I \in N_{G} \cup\{[n]\} .
$$

We describe the map among face lattices $\pi_{P_{B}}: L\left(P e^{n-1}\right) \rightarrow L\left(P_{B}\right)$ given by Definition 2.2 . 
Lemma 5.1 For a face $G$ of a nestohedron $P_{B}$ and a flag of subsets $\mathcal{F}$ on $[n]$ we have $\pi_{P_{B}}(\mathcal{F})=G$ if and only if a flag $\mathcal{F}$ extends the preorder $\preccurlyeq G$.

Proof The proof follows from the coordinate descriptions of corresponding normal cones. The normal cone $\sigma_{G}$ at the face $G$ is given by system of inequalities

$$
\sigma_{G}: x_{i} \leq x_{j} \text { if and only if } i \preccurlyeq{ }_{G} j \text { for } i, j \in[n] \text {. }
$$

The braid cone $\sigma_{\mathcal{F}}$ corresponding to a flag $\mathcal{F}$ is given by relations (2.2). We see that $\sigma_{\mathcal{F}}^{\circ} \subset \sigma_{G}^{\circ}$ if and only if $\mathcal{F}$ extends $\preccurlyeq G$.

For a connected building set $B$ on $[n]$ and a flag of subsets $\mathcal{L}: \emptyset=I_{0} \subset I_{1} \subset$ $\ldots \subset I_{k}=[n]$ denote by $B / \mathcal{L}$ the quotient building set

$$
B / \mathcal{L}=\bigoplus_{j=1}^{k}\left(\left.B\right|_{I_{j}} / I_{j-1}\right)
$$

Define the rank of $\mathcal{L}$ according to $B$ as

$$
\operatorname{rk}_{B}(\mathcal{L})=\sum_{j=1}^{k} \operatorname{rk}\left(\left.B\right|_{I_{j}} / I_{j-1}\right)
$$

Note that $\operatorname{rk}_{B}(\mathcal{L})=n-c(B / \mathcal{L})$, where $c(B / \mathcal{L})$ is the number of components of the quotient building set $B / \mathcal{L}$.

Proposition 5.2 For a connected building set $B$ on $[n]$ and a flag $\mathcal{L}$ of subsets of $[n]$ the ranks $\operatorname{rk}_{P_{B}}(\mathcal{L})$ and $\operatorname{rk}_{B}(\mathcal{L})$ coincide

$$
\operatorname{rk}_{P_{B}}(\mathcal{L})=\operatorname{rk}_{B}(\mathcal{L})
$$

Proof Let $G$ be a proper face of $P_{B}$ of dimension $\operatorname{dim} G=m$ and $\mathcal{L}: \varnothing=I_{0} \subset$ $I_{1} \subset \ldots \subset I_{k}=[n]$ be a flag of subsets of $[n]$ such that $\pi_{P_{B}}(\mathcal{L})=G$. To the face $G$ corresponds the nested set $N_{G}=\left\{J_{1}, \ldots, J_{n-m-1}\right\} \subset B \backslash\{[n]\}$. A simple argumentation shows that

$$
c\left(\left.B\right|_{I_{p}} / I_{p-1}\right)=\left|\left\{J \in N_{G} \cup\{[n]\} \mid J^{\text {root }} \subset I_{p} \backslash I_{p-1}\right\}\right| \text { for all } 1 \leq p \leq k .
$$

Since for any $J \in N_{G} \cup\{[n]\}$ there is a unique $p$ such that $J^{\text {root }} \subset I_{p} \backslash I_{p-1}$ we have

$$
\sum_{j=1}^{k} c\left(\left.B\right|_{I_{j}} / I_{j-1}\right)=n-m .
$$

The following theorem gives an algebraic characterization of the quasisymmetric enumerator function $F_{q}\left(P_{B}\right)$. 
Theorem 5.3 For a building set $B$ the quasisymmetric enumerator function $F_{q}\left(P_{B}\right)$ associated with a nestohedron $P_{B}$ coincides with the value of a universal morphism

$$
F_{q}\left(P_{B}\right)=\Psi_{q}([B]) .
$$

Proof The morphism $\Psi_{q}$ is given by

$$
\Psi_{q}([B])=\sum_{\alpha=\operatorname{gr}(B)}\left(\zeta_{q}\right)_{\alpha}(B) M_{\alpha} .
$$

By Theorem 3.1 the coefficient corresponding to a composition $\alpha=\left(i_{1}, \ldots, i_{k}\right)=n$ is determined by

$$
\left(\zeta_{q}\right)_{\alpha}(B)=\sum_{\mathcal{L}: \operatorname{type}(\mathcal{L})=\alpha} \prod_{j=1}^{k} q^{\operatorname{rk}\left(\left.B\right|_{I_{j}} / I_{j-1}\right)}=\sum_{\mathcal{L}: \operatorname{type}(\mathcal{L})=\alpha} q^{\mathrm{rk}_{B}(\mathcal{L})},
$$

where the sum is over all chains $\mathcal{L}: \varnothing \subset I_{1} \subset \ldots \subset I_{k}=V$ of the type $\alpha$ and $\operatorname{rk}_{B}(\mathcal{L})$ is the rank of the flag $\mathcal{L}$ according to $B$. Thus

$$
F_{q}\left(P_{B}\right)=\sum_{\mathcal{L}} q^{\mathrm{rk}_{B}(\mathcal{L})} M_{\text {type }(\mathcal{L})}
$$

where the sum is over all chains of the ground set $[n]$. Theorem follows according to the expansion (4.1) and Proposition 5.2.

Example The permutohedron $P e^{n-1}=P_{B_{n}}$ is realized as the nestohedron corresponding to the family $B_{n}$ of all subsets of $[n]$. Since $\operatorname{rk}_{B_{n}}(\mathcal{L})=n-|\mathcal{L}|$ for any chain $\mathcal{L}$ of subsets of $[n]$ we have by $(5.3)$ that

$$
F_{q}\left(P e^{n-1}\right)=\sum_{\mathcal{L}} q^{n-|\mathcal{L}|} M_{\operatorname{type}(\mathcal{L})}=\sum_{\alpha=n}\left(\begin{array}{l}
n \\
\alpha
\end{array}\right) q^{n-k(\alpha)} M_{\alpha} .
$$

Consequently by Theorem 4.4 we derive the well known fact

$$
f\left(P e^{n-1}, q\right)=\sum_{\alpha \models n}\left(\begin{array}{l}
n \\
\alpha
\end{array}\right) q^{n-k(\alpha)}
$$

Example For the building set $B=\{\{1\}, \ldots,\{n\},[n]\}$ on $[n]$ the corresponding nestohedron is the $(n-1)$-simplex $P_{B}=\Delta^{n-1}$. Let $\mathcal{L}$ be a chain of the type type $(\mathcal{L})=\alpha \models n$. Obviously $\operatorname{rk}_{B}(\mathcal{L})=l(\alpha)-1$, where $l(\alpha)$ denotes the last component of the composition $\alpha \models n$. Therefore, by (5.2), we have $\left(\zeta_{q}\right)_{\alpha}(B)=\left(\begin{array}{l}n \\ \alpha\end{array}\right) q^{l(\alpha)-1}$, and consequently

$$
F_{q}\left(\Delta^{n-1}\right)=\sum_{\alpha \models n}\left(\begin{array}{l}
n \\
\alpha
\end{array}\right) q^{l(\alpha)-1} M_{\alpha}
$$


By rearranging summands according to last components of compositions we obtain

$$
F_{q}(B)=\sum_{k=1}^{n}\left(\begin{array}{l}
n \\
k
\end{array}\right) q^{k-1} \sum_{\alpha \in=n-k}\left(\begin{array}{c}
n-k \\
\alpha
\end{array}\right) M_{(\alpha, k)}
$$

Taking into account that $M_{(1)}^{n}=\sum_{\alpha \models n}\left(\begin{array}{l}n \\ \alpha\end{array}\right) M_{\alpha}$, for each $n$ we have

$$
F_{q}\left(\Delta^{n-1}\right)=\sum_{k=1}^{n}\left(\begin{array}{l}
n \\
k
\end{array}\right) q^{k-1}\left(M_{(1)}^{n-k}\right)_{k}
$$

Theorem 4.4 gives the expected

$$
f\left(\Delta^{n-1}, q\right)=\sum_{k=1}^{n}\left(\begin{array}{l}
n \\
k
\end{array}\right) q^{k-1}=\frac{(1+q)^{n}-1}{q} .
$$

\subsection{Recursive behavior of $F_{q}\left(P_{B}\right)$}

For a composition $\alpha=\left(a_{1}, \ldots, a_{k}\right)$ and a positive integer $r$ let $(\alpha, r)=$ $\left(a_{1}, \ldots, a_{k}, r\right)$. Define a shifting operator $F \mapsto(F)_{r}$ on $Q S y m$ as the linear extension of the map given on the monomial basis by $M_{\alpha} \mapsto M_{(\alpha, r)}$. Specially $\left(M_{\emptyset}\right)_{r}=M_{(r)}=x_{1}^{r}+x_{2}^{r}+\cdots$.

The next theorem shows that the weighted quasisymmetric enumerator function for nestohedra $F_{q}\left(P_{B}\right)$ is determined by recurrence relations.

Theorem 5.4 The quasisymmetric function $F_{q}(B)=F_{q}\left(P_{B}\right)$ is determined by the following recurrence relations

(1) $F_{q}(\bullet)=M_{(1)}$ for the singleton $\bullet=\{\{1\}\}$.

(2) If $B=B_{1} \sqcup B_{2}$ then $F_{q}(B)=F_{q}\left(B_{1}\right) F_{q}\left(B_{2}\right)$.

(3) If $B$ is connected then $F_{q}(B)=\sum_{I \nsubseteq[n]} q^{n-|I|-1}\left(F_{q}\left(\left.B\right|_{I}\right)\right)_{n-|I|}$.

Proof The assertions (1) and (2) are direct consequences of definition of the enumerator $F_{q}(B)$. It remains to prove the assertion (3). Note that for connected $B$ the contraction $B / I$ remains connected for each $I \subset[n]$ and $\operatorname{rk}(B / I)=n-|I|-1$. If we rearrange the sum in the expansion (5.3) according to predecessors of the maximal elements in chains we obtain

$$
F_{q}(B)=\sum_{I \subsetneq[n]} q^{n-|I|-1} \sum_{\mathcal{L}_{I}} q^{\mathrm{rk}_{\left.B\right|_{I}}\left(\mathcal{L}_{I}\right)} M_{\left(\operatorname{type}\left(\mathcal{L}_{I}\right), n-|I|\right)},
$$

where the last sum is over all chains $\mathcal{L}_{I}$ of $I$. This leads, by repeated application of equation (5.3), to the needed identity.

The recursive behavior of the enumerator $F_{q}\left(P_{B}\right)$ together with Theorem 4.4 reproves the recursive behavior of $f$-polynomials of nestohedra. 
Theorem 5.5 ([17], Theorem 7.11) The $f$-polynomial $f(B, q)$ of a nestohedron $P_{B}$ is determined by the following recurrence relations

(1) $f(\bullet, q)=1$ for the singleton $\bullet=\{\{1\}\}$.

(2) If $B=B_{1} \sqcup B_{2}$ then $f(B, q)=f\left(B_{1}, q\right) f\left(B_{2}, q\right)$.

(3) If $B$ is connected then $f(B, q)=\sum_{I \varsubsetneqq[n]} q^{n-|I|-1} f\left(\left.B\right|_{I}, q\right)$.

\section{Graph-associahedra}

A special class of building sets is produced by simple graphs. The graphical building set $B(\Gamma)$ on a graph $\Gamma$ is the collection of all subsets of vertices such that induced subgraphs are connected. The polytope $P_{\Gamma}=P_{B(\Gamma)}$ is called a graph-associahedron. This class of polytopes, which generalizes the Stasheff associahedron, is introduced by Carr and Devadoss in [5].

In [12], the following Hopf algebra of graphs is considered. Let $\mathcal{G}$ be a vector space over the field $\mathbf{k}$ spanned by isomorphism classes of simple graphs. It is endowed with a Hopf algebra structure by operations

$$
\left[\Gamma_{1}\right] \cdot\left[\Gamma_{2}\right]=\left[\Gamma_{1} \sqcup \Gamma_{2}\right] \text { and } \Delta([\Gamma])=\sum_{I \subset V}\left[\left.\Gamma\right|_{I}\right] \otimes[\Gamma / I],
$$

where $\left.\Gamma\right|_{I}$ is the induced subgraph on $I$ and $\Gamma / I$ is the induced subgraph on $V \backslash I$ with additional edges $u v$ for all pairs of vertices $u, v \notin I$ connected by edge paths through $I$. The correspondence $\Gamma \mapsto B(\Gamma)$ defines a Hopf monomorphism from $\mathcal{G}$ to $\mathcal{B}$.

The induced character on $\mathcal{G}$ over the field of rational functions $\mathbf{k}(q)$ is given by $\zeta_{q}(\Gamma)=q^{\operatorname{rk}(\Gamma)}$, where $\operatorname{rk}(\Gamma)=\operatorname{gr}(\Gamma)-c(\Gamma)$ with $\operatorname{gr}(\Gamma)$ and $c(\Gamma)$ being the numbers of vertices and connected components of the graph $\Gamma$. This results in a $q$-analogue $F_{q}(\Gamma)$ of the quasisymmetric function invariant $F(\Gamma)=F\left(P_{\Gamma}\right)$ of the graph $\Gamma$ introduced and studied in [12]. By formula (5.3) the quasisymmetric function $F_{q}(\Gamma)$ is described by

$$
F_{q}(\Gamma)=\sum_{\mathcal{L}} q^{\mathrm{rk}_{\Gamma}(\mathcal{L})} M_{\text {type }(\mathcal{L})}
$$

where $\operatorname{rk}_{\Gamma}(\mathcal{L})=\sum_{j=1}^{k} \operatorname{rk}\left(\left.\Gamma\right|_{I_{j}} / I_{j-1}\right)$ for a chain $\mathcal{L}: \emptyset \subset I_{1} \subset \cdots \subset I_{k}=V$.

Theorem 5.4 applied on the case of graph-associahedra gives the following recurrence behavior of $F_{q}(\Gamma)$.

Proposition 6.1 The quasisymmetric function $F_{q}(\Gamma)$ associated with a simple graph $\Gamma$ satisfies

(1) $F_{q}(\bullet)=M_{(1)}$ for the one-vertex graph,

(2) If $\Gamma=\Gamma_{1} \sqcup \Gamma_{2}$ then $F_{q}(\Gamma)=F_{q}\left(\Gamma_{1}\right) F_{q}\left(\Gamma_{2}\right)$,

(3) If $\Gamma$ is connected then $F_{q}(\Gamma)=\sum_{I \varsubsetneqq V} q^{|V|-|I|-1}\left(F_{q}\left(\left.\Gamma\right|_{I}\right)\right)_{|V|-|I|}$.

Proposition 6.1 includes the recurrence formula for the special case $q=0$ obtained in [12, Theorem 7.4] 
Corollary 6.2 The quasisymmetric graph invariant $F(\Gamma)$ satisfies

(1) $F(\bullet)=M_{(1)}$ for the one-vertex graph,

(2) If $\Gamma=\Gamma_{1} \sqcup \Gamma_{2}$ then $F(\Gamma)=F\left(\Gamma_{1}\right) F\left(\Gamma_{2}\right)$,

(3) If $\Gamma$ is connected then $F(\Gamma)=\sum_{v \in V}\left(F_{\Gamma \backslash v}\right)_{1}$, where $\Gamma \backslash v$ is the induced graph on $V \backslash\{v\}$.

\subsection{Graphs with the same weighted enumerator $F_{q}(\Gamma)$}

The following nonisomorphic graphs on six vertices with the same quasisymmetric invariant $F(\Gamma)$ were found in [12, Example 7.5], see Fig. 1. We obtain from Corollary 6.2

$$
F\left(\Gamma_{1}\right)=F\left(\Gamma_{2}\right)=720 M_{(1,1,1,1,1,1)}+96 M_{(2,1,1,1,1)}+24 M_{(1,2,1,1,1)} .
$$

By applying Proposition 6.1, we obtain that $F_{q}(\Gamma)$ of these graphs are also equal. In Table 1 are given coefficients of $F_{q}\left(\Gamma_{1}\right)=F_{q}\left(\Gamma_{2}\right)$ by the monomials $q^{k}, k=$ $0,1,2,3,4,5$.

Fig. 1 Graphs with $F\left(\Gamma_{1}\right)=F\left(\Gamma_{2}\right)$
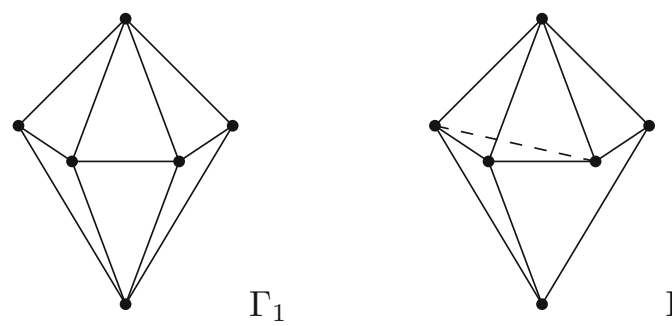

$\Gamma_{2}$

Table $1 \quad F_{q}\left(\Gamma_{1}\right)=F_{q}\left(\Gamma_{2}\right)$

\begin{tabular}{ll}
\hline$q^{5}$ & $M_{(6)}$ \\
\hline$q^{4}$ & $6 M_{(1,5)}+6 M_{(5,1)}+11 M_{(2,4)}+15 M_{(4,2)}+18 M_{(3,3)}$ \\
$q^{3}$ & $30 M_{(1,1,4)}+30 M_{(1,4,1)}+30 M_{(4,1,1)}+66 M_{(2,2,2)}$ \\
& $+4 M_{(2,4)}+2 M_{(3,3)}+56 M_{(1,2,3)}+60 M_{(1,3,2)}+$ \\
& $+44 M_{(2,1,3)}+44 M_{(2,3,1)}+54 M_{(3,1,2)}+54 M_{(3,2,1)}$ \\
& $108 M_{(3,1,1,1)}+120 M_{(1,3,1,1)}+120 M_{(1,1,3,1)}+$ \\
$q^{2}$ & $+120 M_{(1,1,1,3)}+180 M_{(1,1,2,2)}+168 M_{(1,2,1,2)}+$ \\
& $+168 M_{(1,2,2,1)}+132 M_{(2,1,1,2)}+132 M_{(2,1,2,1)}+$ \\
& $+132 M_{(2,2,1,1)}+4 M_{(1,2,3)}+16 M_{(2,1,3)}+$ \\
& $+16 M_{(2,3,1)}+6 M_{(3,1,2)}+6 M_{(3,2,1)}+24 M_{(2,2,2)}$ \\
& $360 M_{(1,1,1,1,2)}+360 M_{(1,1,1,2,1)}+360 M_{(1,1,2,1,1)}+$ \\
& $+336 M_{(1,2,1,1,1,)}+264 M_{(2,1,1,1,1)}+12 M_{(1,2,1,2)}+$ \\
$q^{1}$ & $+12 M_{(1,2,2,1)}+48 M_{(2,1,1,2)}+48 M_{(2,1,2,1)}+$ \\
& $+48 M_{(2,2,1,1)}+12 M_{(3,1,1,1)}$ \\
& $720 M_{(1,1,1,1,1)}+96 M_{(2,1,1,1,1)}+24 M_{(1,2,1,1,1)}$ \\
&
\end{tabular}


Table 2 Vertex degrees of $G_{1}$

\begin{tabular}{llllllllllll}
\hline $\mathrm{d}$ & 30 & 29 & 28 & 26 & 25 & 16 & 15 & 14 & 13 & 12 & 11 \\
$\mathrm{n}$ & 4 & 2 & 2 & 2 & 2 & 9 & 9 & 3 & 4 & 6 & 13 \\
\hline
\end{tabular}

Table 3 Vertex degrees of $G_{2}$

\begin{tabular}{lllllllllll}
\hline $\mathrm{d}$ & 30 & 29 & 28 & 26 & 16 & 15 & 14 & 13 & 12 & 11 \\
$\mathrm{n}$ & 2 & 4 & 2 & 4 & 10 & 6 & 4 & 6 & 6 & 12 \\
\hline
\end{tabular}

Theorem 4.4 then implies that $f$-polynomials of corresponding graph-associahedra are equal. We found

$$
f\left(P_{\Gamma_{1}}, q\right)=f\left(P_{\Gamma_{2}}, q\right)=q^{5}+56 q^{4}+462 q^{3}+1308 q^{2}+1500 q+600 .
$$

Nevertheless the polytopes $P_{\Gamma_{i}}, i=1,2$ are not combinatorially equivalent. We can show this by looking at the 1 -skeletons of dual polytopes $G_{i}=\left(\left(P_{\Gamma_{i}}\right)^{*}\right)^{(1)}, i=1,2$. These graphs have different sequences of vertex degrees, see Tables 2 and 3 . Here, $d$ stands for the vertex degree and $n$ for the number of vertices with this degree. Recall that dual polytopes of nestohedra are represented by nested set complexes, see [18, Theorem 6.5]. Therefore $G_{i}, i=1,2$ are graphs whose vertices correspond to connected induced subgraphs of $\Gamma_{i}, i=1,2$ and edges are given by pairs of connected induced subgraphs $\left\{H_{1}, H_{2}\right\}$ such that either one is contained into another or their union is not connected.

Remark 6.3 By using MatLab program, we found exactly three pairs of six-vertex graphs with the same quasisymmetric function $F(\Gamma)$. If we label adequately the vertices then the sets of edges of graphs given in Fig. 1 are $E\left(\Gamma_{1}\right)=$ $\{12,13,14,15,23,34,45,26,36,46,56\}$ and $E\left(\Gamma_{2}\right)=\{12,13,14,15,23,24,34$, $45,26,36,56\}$. Two other pairs are obtained by deleting edges 14 and 14, 26 from $\Gamma_{1}$ and $\Gamma_{2}$, respectively. Similarly as for the pair $\Gamma_{1}$ and $\Gamma_{2}$, it can be shown that the other two pairs have equal $q$-analogues $F_{q}(\Gamma)$ and henceforth equal $f$-polynomials of corresponding $P_{\Gamma}$, which are also combinatorially nonequivalent polytopes.

\section{Matroid base polytope}

A standard reference monograph for matroid theory is [16]. The most adequate for our purposes is a definition of a matroid as a collection of bases. A matroid $M=([n], \mathcal{B})$ on the ground set $[n]$ is a nonempty collection $\mathcal{B}$ of subsets of $[n]$ such that the exchange property is satisfied:

- For each $B_{1}, B_{2} \in \mathcal{B}$ and $i \in B_{1} \backslash B_{2}$ there is $j \in B_{2} \backslash B_{1}$ such that $\left(B_{1} \backslash\{i\}\right) \cup\{j\} \in$ $\mathcal{B}$.

The members of $\mathcal{B}$ are called bases of $M$ and we denote the collection of bases of $M$ by $\mathcal{B}(M)$. All bases have the same number of elements which is called the $\operatorname{rank} r(M)$ of the matroid $M$. 
Definition 7.1 The matroid base polytope is a convex polytope

$$
P_{M}=\operatorname{Conv}\left\{e_{B} \mid B \in \mathcal{B}(M)\right\}
$$

where $e_{B}=\sum_{i \in B} e_{i}$ and $e_{i}, i=1, \ldots, n$ are the standard basis vectors in $\mathbb{R}^{n}$.

The polytope $P_{M}$ is contained in the hypersimplex

$$
\Delta_{r}^{n}=[0,1]^{n} \cap\left\{x_{1}+x_{2}+\cdots+x_{n}=r(M)\right\} .
$$

The following characterization of matroids in terms of their base polytopes shows that matroid base polytopes are generalized permutohedra.

Theorem 7.2 ([9], Section 2.2, Theorem 1 and [10], Theorem 4.1) Let $\mathcal{S}$ be a collection of $r$-subsets of $[n]$ and $P_{\mathcal{S}}$ be a convex polytope in $\mathbb{R}^{n}$

$$
P_{\mathcal{S}}=\operatorname{Conv}\left\{e_{S} \mid S \in \mathcal{S}\right\},
$$

where $e_{S}=\sum_{i \in S} e_{i}$. Then $\mathcal{S}$ is the collection of bases of a matroid if and only if every edge of $P_{\mathcal{S}}$ is a translate of the vector $e_{i}-e_{j}$, for some $i, j \in[n]$.

Definition 7.3 For a matroid $M=([n], \mathcal{B})$ the elements $i, j \in[n]$ are equivalent if there exist bases $B_{1}$ and $B_{2}$ with $i \in B_{1}$ and $B_{2}=\left(B_{1} \backslash\{i\}\right) \cup\{j\}$. The equivalence classes are called connected components of $M$. The matroid $M$ is connected if it has only one connected component.

Denote by $c(M)$ the number of connected components of a matroid $M$.

Proposition 7.4 ([8], Proposition 2.4) The dimension of the matroid base polytope $P_{M}$ corresponding to a matroid $M$ on $[n]$ is determined by

$$
\operatorname{dim}\left(P_{M}\right)=n-c(M)
$$

\subsection{Flags of matroid base polytope}

For a vector $\omega=\left(\omega_{1}, \omega_{2}, \ldots, \omega_{n}\right) \in \mathbb{Z}_{+}^{n}$ we regard the function $\omega^{*}$ on the matroid base polytope $P_{M}$ as a weight function on a matroid $M$, so that the $\omega$-weight of a basis $B=\left\{i_{1}, \ldots, i_{r(M)}\right\} \in \mathcal{B}(M)$ is given by

$$
\omega^{*}(B)=\left\langle\omega, e_{B}\right\rangle=\omega_{i_{1}}+\cdots+\omega_{i_{r(M)}} .
$$

Let $\mathcal{B}_{\omega} \subset \mathcal{B}(M)$ be the collection of bases of $M$ having maximum $\omega$-weight. The collection $\mathcal{B}_{\omega}$ consists of all bases obtained by greedy algorithm. The polytope $P_{\mathcal{B}_{\omega}}$ is the face of $P_{M}$ on which $\omega^{*}$ is maximized. Theorem 7.2 implies that $\mathcal{B}_{\omega}$ is the collection of bases of a matroid which we denote by $M_{\omega}$. 
Recall that $\omega$ determines a unique flag of subsets

$$
\mathcal{F}_{\omega}: \emptyset=F_{0} \subset F_{1} \subset \ldots \subset F_{k} \subset F_{k+1}=[n]
$$

for which $\omega$ is constant on $F_{i} \backslash F_{i-1}, i=1, \ldots, k+1$ and satisfies $\left.\omega\right|_{F_{i} \backslash F_{i-1}}<$ $\left.\omega\right|_{F_{i+1} \backslash F_{i}}, i=1, \ldots k$. The following proposition shows that $M_{\omega}$ depends only on the flag $\mathcal{F}_{\omega}$. By $r(A)=r\left(\left.M\right|_{A}\right)$, where $\left.M\right|_{A}$ is the restriction of $M$ to $A \subset[n]$, is defined the rank function of $M$.

Proposition 7.5 ([3], Proposition 1) Let $\omega \in \mathbb{Z}_{+}^{n}$ and $\mathcal{F}=\mathcal{F}_{\omega}$ is the corresponding flag given by (7.1). A base $B \in \mathcal{B}(M)$ of a matroid $M$ has the maximum $\omega$-weight, i.e. $B \in B_{\omega}$ if and only if $\left|B \cap\left(F_{i} \backslash F_{i-1}\right)\right|=r\left(F_{i-1}^{c}\right)-r\left(F_{i}^{c}\right)$, where $F_{i}^{c}=[n] \backslash F_{i}, i=$ $1, \ldots, k+1$.

We say that a flag $\mathcal{F}$ is maximized at a base $B \in \mathcal{B}(M)$ if and only if $B \in B_{\omega}$ and $\mathcal{F}=\mathcal{F}_{\omega}$ for some $\omega \in \mathbb{Z}_{+}^{n}$. Denote by $M / \mathcal{F}$ the matroid $M_{\omega}$ corresponding to a flag $\mathcal{F}=\mathcal{F}_{\omega}$. We determine the map $\pi_{P_{M}}: L\left(P e^{n-1}\right) \rightarrow L\left(P_{M}\right)$ associated with the matroid base polytope by Definition 2.2, namely

$$
\pi_{P_{M}}(\mathcal{F})=P_{M / \mathcal{F}}
$$

The following proposition describes the matroid $M / \mathcal{F}$ as a sum of its minors.

Proposition 7.6 ([3], Proposition 2) If $\mathcal{F}=\left\{\emptyset=F_{0} \subset \ldots \subset F_{k+1}=[n]\right\}$, then

$$
M / \mathcal{F}=\bigoplus_{i=1}^{k+1}\left(\left.M\right|_{F_{i-1}^{c}}\right) / F_{i}^{c}
$$

Since for the direct sum of matroids we have $P_{M_{1} \oplus M_{2}}=P_{M_{1}} \times P_{M_{2}}$, by Proposition 7.4 we obtain

Corollary 7.7 The $P_{M}$-rank of a flag $\mathcal{F}$ is given by

$$
\operatorname{rk}_{P_{M}}(\mathcal{F})=\operatorname{dim}\left(P_{M / \mathcal{F}}\right)=n-\sum_{i=1}^{k+1} c\left(\left(\left.M\right|_{F_{i-1}^{c}}\right) / F_{i}^{c}\right)
$$

\subsection{Quasisymmetric enumerator $F_{q}\left(P_{M}\right)$ for matroids}

The Hopf algebra of matroids Mat was introduced by Schmitt [19] and more intensively studied by Crapo and Schmitt [6,7].

As a vector space $\mathcal{M}$ at is linearly spanned over the field $\mathbf{k}$ by isomorphism classes of finite matroids $[M]$. The direct sum induces a product, while a coproduct is determined by restrictions and contractions

$$
\left[M_{1}\right] \cdot\left[M_{2}\right]=\left[M_{1} \oplus M_{2}\right] \quad \text { and } \quad \Delta[M]=\sum_{A \subseteq E}\left[\left.M\right|_{A}\right] \otimes[M / A]
$$


The Hopf algebra Mat is graded Mat $=\bigoplus_{n \geq 0} \mathcal{M a t}_{n}$, where $\mathcal{M a t}_{n}$ denotes the subspace spanned by elements $[M]$ for which the ground set $E$ has cardinality $n$. The Hopf algebra $\mathcal{M}$ at is graded, connected, commutative, but non-cocommutative. The unit is the class of a unique matroid on the empty set $\left[M_{\emptyset}\right]$.

The character $c:$ Mat $\rightarrow \mathbf{k}$ defined by

$$
c([M])=\left\{\begin{array}{l}
1, M \text { is a direct sum of loops and isthmuses, } \\
0, \text { otherwise }
\end{array}\right.
$$

turns Mat into a combinatorial Hopf algebra considered by Billera, Jia and Reiner in [4]. By Theorem 3.1, there is a unique morphism $\Psi:(\mathcal{M a t}, c) \rightarrow\left(Q S y m, \zeta_{\mathcal{Q}}\right)$ of combinatorial Hopf algebras given in the monomial basis by

$$
\Psi([M])=\sum_{\alpha \models n} c_{\alpha}([M]) M_{\alpha}
$$

Proposition 7.8 ([4], Proposition 3.3) The coefficient $c_{\alpha}([M])$ is the number of flags $\mathcal{F}: \emptyset=F_{0} \subset \cdots \subset F_{k+1}=[n]$ having type $(\mathcal{F})=\alpha$ and for which each subquotient $\left(\left.M\right|_{F_{i}}\right) / F_{i-1}$ is a direct sum of loops and isthmuses.

We extend the basic field $\mathbf{k}$ into the field of rational functions $\mathbf{k}(q)$ and define a character $c_{q}:$ Mat $\rightarrow \mathbf{k}(q)$ with

$$
c_{q}([M])=q^{\mathrm{rk}(M)},
$$

where $\operatorname{rk}(M)=n-c(M)$. Let $\Psi_{q}:\left(\mathcal{M a t}, c_{q}\right) \rightarrow\left(Q S y m, \zeta_{\mathcal{Q}}\right)$ be a unique morphism of combinatorial Hopf algebras over $\mathbf{k}(q)$ and rev : QSym $\rightarrow$ Sym be a linear involution defined on the monomial basis by $\operatorname{rev}\left(M_{\alpha}\right)=M_{\operatorname{rev}(\alpha)}$.

Theorem 7.9 For a matroid $M$ the quasisymmetric enumerator function $F_{q}\left(P_{M}\right)$ associated with the matroid base polytope $P_{M}$ and the reverse of $\Psi_{q}([M])$ coincide

$$
F_{q}\left(P_{M}\right)=\operatorname{rev}\left(\Psi_{q}([M])\right)
$$

Proof The morphism $\Psi_{q}$ is given by

$$
\Psi_{q}([M])=\sum_{\alpha \models n} c_{q, \alpha}([M]) M_{\alpha}
$$

where

$$
c_{q, \alpha}([M])=\sum_{\mathcal{F}: \operatorname{type}(\mathcal{F})=\alpha} \prod_{i=1}^{k} q^{\operatorname{rk}\left(\left(\left.M\right|_{F_{i}}\right) / F_{i-1}\right)} .
$$


By Corollary 7.7 we have

$$
c_{q, \alpha}([M])=\sum_{\mathcal{F}: \operatorname{type}(\mathcal{F})=\alpha} q^{\mathrm{rk}_{P_{M}}\left(\mathcal{F}^{o p}\right)} .
$$

Consequently,

$$
\Psi_{q}([M])=\sum_{\mathcal{F}} q^{\mathrm{rk}_{P_{M}}\left(\mathcal{F}^{o p}\right)} M_{\text {type }(\mathcal{F})},
$$

where the sum is over all flags of $[n]$. Hence, from (4.3), we obtain the form of $F_{q}\left(P_{M}\right)$ given by (4.1).

The reverse involution does not affect on the principal specialization $\mathbf{p s}\left(M_{\alpha}\right)(m)=$ $\operatorname{ps}\left(M_{\operatorname{rev}(\alpha)}\right)(m)$. As a consequence of Theorem 7.9 and Theorem 4.4, we obtain an algebraic description of the $f$-polynomials of matroid base polytopes.

Theorem 7.10 The $f$-polynomial of a matroid base polytope $P_{M}$ is given by

$$
f\left(P_{M}, q\right)=(-1)^{n} \mathbf{p s}\left(\Psi_{-q}([M])\right)(-1) .
$$

\subsection{The uniform matroid base polytope}

We use Theorem 7.10 to calculate $f$-polynomials of uniform matroid base polytopes $P_{U_{n, r}}$. The uniform matroid $U_{n, r}$ is a matroid with the set of bases $\mathcal{B}\left(U_{n, r}\right)=\left(\begin{array}{c}{[n]} \\ r\end{array}\right)$ consisting of all $r$-elements subsets of $[n]$. From definition, it follows that $P_{U_{n, r}}$ is the hypersimplex

$$
P_{U_{n, r}}=\Delta_{r}^{n}
$$

Note that $\mathcal{B}\left(U_{n, 0}\right)=\{\emptyset\}$ and $\mathcal{B}\left(U_{n, n}\right)=\{[n]\}$, so $P_{U_{n, 0}}=\mathbf{0}$ and $P_{U_{n, n}}=\mathbf{1}$ are single points, where $\mathbf{0}=(0, \ldots, 0)$ and $\mathbf{1}=(1, \ldots, 1)$.

Consider the uniform matroid $U_{n, r}$ and assume that $0<r<n$. Let $\mathcal{F}: \emptyset=$ $F_{0} \subset F_{1} \subset \ldots \subset F_{k+1}=[n]$ be a flag with $\operatorname{type}(\mathcal{F})=\left(\alpha_{1}, \ldots, \alpha_{k+1}\right)$. Define $i_{0}=i_{0}(\mathcal{F})=\min \left\{i \mid \alpha_{1}+\cdots+\alpha_{i} \geq r\right\}$ and partial sums $r^{\prime}=\alpha_{1}+\cdots+\alpha_{i_{0}-1}, r^{\prime \prime}=$ $r^{\prime}+\alpha_{i_{0}}$. By Proposition 7.6 we find

$$
M / \mathcal{F}=U_{r^{\prime}, r^{\prime}} \oplus U_{\alpha_{i_{0}}, r-r^{\prime}} \oplus U_{n-r^{\prime \prime}, 0}
$$

It shows that faces of the uniform matroid base polytopes are uniform matroid base polytopes as well, the fact that is obvious from the description by hypersimplices. We have $P_{M / \mathcal{F}} \cong P_{U_{\alpha_{i}}, r-r^{\prime}}$, which implies the following formula for the $P_{M}$-rank, where $M=U_{n, r}$

$$
\operatorname{rk}_{P_{M}}(\mathcal{F})=\operatorname{dim}\left(P_{M / \mathcal{F}}\right)=\left\{\begin{array}{ll}
0, & r=r^{\prime \prime} \\
\alpha_{i_{0}}-1, & r<r^{\prime \prime}
\end{array} .\right.
$$


Let $\circ$ be the concatenation product defined on the monomial basis by

$$
M_{\alpha} \circ M_{\beta}=M_{\alpha \beta}
$$

and linearly extended to the algebra of quasisymmetric functions. We obtain from (7.2)

$$
\begin{aligned}
F_{q}\left(P_{U_{n, r}}\right)= & \left(\begin{array}{l}
n \\
r
\end{array}\right)\left(M_{1}\right)^{r} \circ\left(M_{1}\right)^{n-r} \\
& +\sum_{0 \leq r^{\prime}<r<r^{\prime}+\lambda \leq n}\left(\begin{array}{c}
n \\
r^{\prime}
\end{array}\right)\left(\begin{array}{c}
n-r^{\prime} \\
\lambda
\end{array}\right) q^{\lambda-1}\left(M_{1}\right)^{r^{\prime}} \circ M_{\lambda} \circ\left(M_{1}\right)^{n-r^{\prime}-\lambda} .
\end{aligned}
$$

The principal specialization evaluated at -1 gives

$$
f\left(P_{U_{n, r}}, q\right)=\left(\begin{array}{l}
n \\
r
\end{array}\right)+\sum_{0 \leq r^{\prime}<r<r^{\prime}+\lambda \leq n}\left(\begin{array}{c}
n \\
r^{\prime}
\end{array}\right)\left(\begin{array}{c}
n-r^{\prime} \\
\lambda
\end{array}\right) q^{\lambda-1} .
$$

It determines the $f$-vector of $P_{U_{n, r}}$ in terms of trinomial coefficients

$$
f_{0}=\left(\begin{array}{l}
n \\
r
\end{array}\right) \text { and } f_{k-1}=\sum_{0 \leq r^{\prime}<r<r^{\prime}+k \leq n}\left(\begin{array}{c}
n \\
r^{\prime}, k, n-r^{\prime}-k
\end{array}\right) \text { for } k=2, \ldots, n
$$

Example The polytope $P_{U_{4,2}}=\Delta_{2}^{4}$ is an octahedron. We obtain

$$
\begin{aligned}
F_{q}\left(P_{U_{4,2}}\right)= & 6\left(M_{(2,2)}+2 M_{(1,1,2)}+2 M_{(2,1,1)}+4 M_{(1,1,1,1)}\right) q^{0}+12 M_{(1,2,1)} q \\
& +4\left(M_{(1,3)}+M_{(3,1)}\right) q^{2}+M_{(4)} q^{3}
\end{aligned}
$$

which yields to the expected expression $f\left(P_{U_{4,2}}, q\right)=6+12 q+8 q^{2}+q^{3}$.

\section{Properties of $F_{q}(M)$}

Further, we will write $F_{q}(M)$ instead of $F_{q}\left(P_{M}\right)$. We examine some algebraic properties of the quasisymmetric enumerator $F_{q}(M)$. First we give an example which shows that $F_{q}(M)$ contains more information about matroids than its specialization at $q=0$. The following example of matroids with the same quasisymmetric invariant $F(M)$ is borrowed from [4, Example 8.1].

Example Let $M_{1}$ and $M_{2}$ be affine matroids depicted on Fig. 2. $M_{1}$ and $M_{2}$ are the rank 3 matroids on the set [6] having every triple but $\{1,2,3\},\{4,5,6\}$ and $\{1,2,3\},\{3,4,5\}$ as bases, respectively. Consider flags $\emptyset \subset\{i\} \subset[6] \backslash\{j\} \subset[6], 1 \leq i \neq j \leq 6$ of the type $(1,4,1)$. All minors $\left.M_{1}\right|_{[6] \backslash\{j\}} /\{i\}$ are connected, so we have the summand $30 q^{3} M_{(1,4,1)}$ in $F_{q}\left(M_{1}\right)$. On the other hand the flag $\emptyset \subset\{3\} \subset\{1,2,3,4,5\} \subset[6]$ produces a two components minor of $M_{2}$ which contributes with $q^{2} M_{(1,4,1)}$ in $F_{q}\left(M_{2}\right)$. It shows that $F_{q}\left(M_{1}\right) \neq F_{q}\left(M_{2}\right)$. 

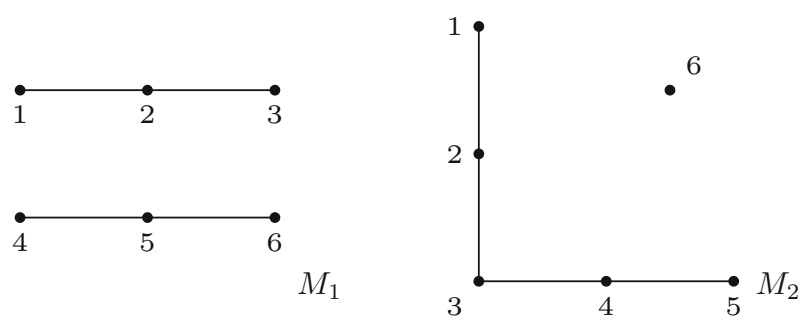

Fig. 2 Matroids with $F\left(M_{1}\right)=F\left(M_{2}\right)$

\subsection{Matroid duality}

For a matroid $M$ on $[n]$ with the collection of bases $\mathcal{B}(M)$ the dual matroid $M^{*}$ is defined by the set of bases $\mathcal{B}\left(M^{*}\right)=\{[n] \backslash B \mid B \in \mathcal{B}(M)\}$. The affine transformation aff $:[0,1]^{n} \rightarrow[0,1]^{n}$ of the $n$-cube aff $(x)=\mathbf{1}-x, x \in[0,1]^{n}$, where $\mathbf{1}=$ $(1, \ldots, 1)$ determines an affine isomorphism between $P_{M}$ and $P_{M^{*}}$. The behavior of the quasisymmetric invariant $F_{q}(M)$ for $q=0$ under matroid duality is determined by [4, Proposition 4.1]. We generalize this to the $q$-analogue $F_{q}(M)$.

Lemma 8.1 For a matroid $M$ and a flag $\mathcal{F}$ on its ground set $[n]$ it holds

$$
\operatorname{aff}\left(P_{M / \mathcal{F}}\right)=P_{M^{*} / \mathcal{F}^{o p}}
$$

Proof The statement follows from the fact that the flag $\mathcal{F}$ is maximized at a base $B \in \mathcal{B}(M)$ if and only if $\mathcal{F}^{o p}$ is maximized at the cobase $[n] \backslash B \in \mathcal{B}\left(M^{*}\right)$.

Proposition 8.2 For a matroid $M$ and its dual $M^{*}$ the corresponding quasisymmetric enumerators are related with

$$
F_{q}\left(M^{*}\right)=\operatorname{rev}\left(F_{q}(M)\right)
$$

Proof We use the expansion (4.2)

$$
F_{q}\left(M^{*}\right)=\sum_{G \in L\left(P_{M^{*}}\right)} q^{\operatorname{dim}(G)} \sum_{\mathcal{F}: \pi_{P_{M^{*}}}(\mathcal{F})=G} M_{\text {type }(\mathcal{F})}
$$

Denote by $G^{*}=\operatorname{aff}(G)$. Then by Lemma 8.1 and the fact $\operatorname{dim}(G)=\operatorname{dim}\left(G^{*}\right)$ we can write

$$
F_{q}\left(M^{*}\right)=\sum_{G^{*} \in L\left(P_{M}\right)} q^{\operatorname{dim}\left(G^{*}\right)} \sum_{\mathcal{F}: \pi_{P_{M}}\left(\mathcal{F}^{o p}\right)=G^{*}} M_{\text {type }\left(\mathcal{F}^{o p}\right)} .
$$

The proposition follows from (4.3). 


\subsection{Valuation}

For matroids $M_{1}=\left([n], \mathcal{B}_{1}\right)$ and $M_{2}=\left([n], \mathcal{B}_{2}\right)$ of the same rank $r$, we can define $M_{1} \cap M_{2}=\left([n], \mathcal{B}_{1} \cap \mathcal{B}_{2}\right)$ which is usually not a matroid, but if polytopes $P_{M_{1}}$ and $P_{M_{2}}$ meet along a common nonempty face, the intersection $M_{1} \cap M_{2}$ will be a matroid of rank $r$ and $P_{M_{1}} \cap P_{M_{2}}=P_{M_{1} \cap M_{2}}$, see [4, Proposition 7.2]. A matroid base polytope decomposition is a decomposition $P_{M}=\sqcup_{i=1}^{k} P_{M_{i}}$ into matroid base polytopes $P_{M_{i}}, i=1, \ldots, k$ such that $P_{M_{i}} \cap P_{M_{j}}$ is a common face of both $P_{M_{i}}$ and $P_{M_{j}}$ for each $i \neq j$. Specially, for $k=2$, a decomposition is called a hyperplane split.

The enumerator $F_{q}(Q)$ for generalized permutohedra lacks to be valuative, contrary to its specialization $q=0$, see [4, Theorem 9.2]. We illustrate this by the following example based on [4, Remark 8.5].

Example Let $M_{1}$ be an affine matroid of rank 3 depicted on Fig. 2 and $M_{2}, M_{3}$ be matroids given by the sets of bases

$$
\begin{aligned}
& \mathcal{B}\left(M_{2}\right)=\{123,124,125,126,134,135,136,234,235,236\}, \\
& \mathcal{B}\left(M_{3}\right)=\{145,146,156,245,246,256,345,346,356,456\} .
\end{aligned}
$$

Then $M_{2} \cong M_{3}, M_{1} \cap M_{2} \cong M_{1} \cap M_{3} \cong U_{3,2} \oplus U_{3,1}$ and we have a decomposition of the hypersimplex $P_{U_{6,3}}=P_{M_{1}} \sqcup P_{M_{2}} \sqcup P_{M_{3}}$. We can check that

$$
F_{q}\left(U_{6,3}\right) \neq F_{q}\left(M_{1}\right)+F_{q}\left(M_{2}\right)+F_{q}\left(M_{3}\right)-F_{q}\left(M_{1} \cap M_{2}\right)-F_{q}\left(M_{1} \cap M_{3}\right) .
$$

\section{Conclusion}

The weighted quasisymmetric enumerator $F_{q}(Q)$ associated with generalized permutohedra has two main meaning. Geometrically, it is the enumerator of lattice points of the normal fan of a generalized permutohedron and this aspect was originated by Billera, Jia and Reiner in [4]. Algebraically, it appears as a result of two universal objects, the first one is the Hopf monoid of generalized permutohedra GP and the second one is the combinatorial Hopf algebra of quasisymmetric functions QSym. This categorical and algebraic approach is originated by Aguiar, Bergeron and Sottile in [2] and more recently by Aguiar and Ardila in [1]. The ubiquity of generalized permutohedra comes from their equivalence with submodular functions. Properly defined submodular functions associated with combinatorial objects give their embeddings as Hopf submonoids to the Hopf monoid GP. Composing with the functor that takes Hopf monoids to Hopf algebras and with universal morphism to quasisymmetric functions produce the invariant $F_{q}(Q)$. Aguiar and Ardila obtained a simple formula for antipode of GP which can help in further study of properties of the enumerator $F_{q}(Q)$. Apart from that we can exploit the idea of universality of the Hopf monoid GP to obtain enumerative invariants of other combinatorial objects such as posets, hypergraphs, simplicial complexes etc. Also it would be interesting to try to extend this lattice points enumerator to other types of generalized Coxeter permutohedra. 


\section{References}

1. Aguiar, M., Ardila, F.: Hopf monoids and generalized permutohedra, arXiv:1709.07504

2. Aguiar, M., Bergeron, N., Sottile, F.: Combinatorial Hopf algebras and generalized Dehn-Sommerville relations. Compos. Math. 142, 1-30 (2006)

3. Ardila, F., Klivans, C.: The Bergman complex of a matroid and phylogenetic trees. J. Combin. Theory Ser. B 96, 38-49 (2006)

4. Billera, L., Jia, N., Reiner, V.: A quasisymmetric function for matroids. European J. Combin. 30, 1727-1757 (2009)

5. Carr, M.P., Devadoss, S.L.: Coxeter complexes and graph-associahedra. Topology Appl. 153(12), 2155-2168 (2006)

6. Crapo, H., Schmitt, W.: A free subalgebra of the algebra of matroids. European J. Combin. 26, 10661085 (2005)

7. Crapo, H., Schmitt, W.: A unique factorization theorem for matroids. J. Combin. Theory Ser. A 112, 222-249 (2005)

8. Feichtner, E.M., Sturmfels, B.: Matroid polytopes, nested sets and Bergman fans. Port. Math. 62(4), 437-468 (2005)

9. Gel'fand, I.M., Serganova, V.V.: Combinatorial geometries and torus strata on homogeneous compact manifolds. Russian Math. Surveys 42(2), 133-168 (1987)

10. Gel'fand, I.M., Goresky, M., MacPherson, R., Serganova, V.: Combinatorial geometries, convex polyhedra, and Schubert cells. Adv. Math. 63, 301-316 (1987)

11. Grinberg, D., Reiner, V.: Hopf algebras in combinatorics, arXiv: 1409.8356

12. Grujić, V.: Quasisymmetric functions for nestohedra. SIAM J. Discrete Math. 31(4), 2570-2585 (2017)

13. Grujić, V.: Counting faces of graphical zonotopes. Ars Math. Contemp. 13, 227-234 (2017)

14. Grujić, V., Stojadinović, T.: Counting faces of nestohedra. Sem. Lothar. Combin. 78B, 17 (2017)

15. Humpert, B., Martin, J.: The incidence Hopf algebra of graphs. SIAM J. Discrete Math. 26(2), 555-570 (2012)

16. Oxley, J.G.: Matroid theory. Oxford University Press, New York (1992)

17. Postnikov, A.: Permutohedra, associahedra, and beyond. Int. Math. Res. Not. IMRN 2009, 1026-1106 (2009)

18. Postnikov, A., Reiner, V., Williams, L.: Faces of generalized permutohedra. Doc. Math. 13, 207-273 (2008)

19. Schmitt, W.R.: Incidence Hopf algebras. J. Pure Appl. Algebra 96(3), 299-330 (1994)

20. Stanley, R.P.: A symmetric function generalization of the chromatic polynomial of a graph. Adv. Math. 111, 166-194 (1995)

Publisher's Note Springer Nature remains neutral with regard to jurisdictional claims in published maps and institutional affiliations. 